\title{
Health-related quality of life and radiographic vertebral fracture
}

\author{
W. Cockerill $\cdot$ M. Lunt $\cdot$ A.J. Silman $\cdot$ C. Cooper \\ P. Lips · A.K. Bhalla $\cdot$ J.B. Cannata $\cdot$ R. Eastell \\ D. Felsenberg · C. Gennari $\cdot$ O. Johnell $\cdot$ J.A. Kanis \\ C. Kiss · P. Masaryk - M. Naves - G. Poor · H. Raspe \\ D.M. Reid $\cdot$ J. Reeve $\cdot$ J. Stepan $\cdot$ C. Todd \\ A.D. Woolf · T.W. O'Neill
}

Received: 26 August 2002/ Accepted: 15 October 2003/Published online: 13 November 2003

(C) International Osteoporosis Foundation and National Osteoporosis Foundation 2003

\begin{abstract}
Background: Vertebral fractures are associated with back pain and disability; however, relatively little is known about the impact of radiographic vertebral fractures on quality of life in population samples. The aim of this study was to determine the impact of a recent radiographic vertebral fracture on health-related quality of life (HRQoL). Methods: Men and women aged 50 years and over were recruited from population registers in 12 European centers. Subjects completed an interviewer-administered questionnaire and had lateral spine radiographs performed. Subjects in these centers were followed prospectively and had repeat spinal radiographs performed a mean of 3.8 years
\end{abstract}

W. Cockerill · M. Lunt · A.J. Silman · T.W. O’Neill (ه)

ARC Epidemiology Research Unit, University of Manchester,

Stopford Building, Manchester, M13 9PT, UK

E-mail: Terry@fsi.ser.man.ac.uk

Tel.: + 44-161-2755040

Fax: +44-161-2755043

C. Cooper

MRC Environmental Epidemiology Unit,

Southampton General Hospital, Southampton, UK

P. Lips

Department of Endocrinology, Academic Hospital,

Vrije Universiteit, Amsterdam, The Netherlands

A.K. Bhalla

Royal National Hospital for Rheumatic Diseases, Bath, UK

J.B. Cannata $\cdot$ M. Naves

Asturia General Hospital, Oviedo, Spain

R. Eastell

Bone Metabolism Group, Northern General Hospital,

Sheffield, UK

D. Felsenberg

Department of Radiology and Nuclear Medicine,

Free University, Berlin, Germany

C. Gennari

Institute of Clinical Medicine, University of Siena, Siena, Italy

O. Johnell

Department of Orthopedics, Lund University, Malmo, Sweden later. Prevalent deformities were defined using established morphometric criteria, and incident vertebral fractures by both morphometric criteria and qualitative assessment. For each incident fracture case, three controls matched for age, gender, and center were selected: one with a prevalent deformity (at baseline) and two without prevalent deformities. All subjects were interviewed or completed a postal questionnaire instrument which included Short Form 12 (SF-12), the EQ-5D (former EuroQol), and the quality of life questionnaire of the International Osteoporosis Foundation (QUALEFFO). The median time from the second spinal radiograph until the quality of life survey was 1.9 years. Comparison between cases and their matched controls was undertaken using the

\section{J. A. Kanis}

Centre for Metabolic Bone Disease, Sheffield, UK

C. Kiss · G. Poor

National Institute of Rheumatology and Physiotherapy,

Budapest, Hungary

P. Masaryk

Institute of Rheumatic Diseases, Piestany, Slovakia

H. Raspe

Institute of Social Medicine, Lubeck, Germany

D. M. Reid

Department of Medicine and Therapeutics,

University of Aberdeen, Aberdeen, UK

J. Reeve

Institute of Public Health, Cambridge, UK

J. Stepan

Institute of Rheumatology,

Charles University, Prague, Czech Republic

C. Todd

Department of Nursing, Midwifery and Health Visiting, University of Manchester, Manchester, UK

A. D. Woolf

Department of Rheumatology,

Royal Cornwall Hospital, Truro, UK 
signed rank test. Results: 73 subjects with incident vertebral fracture (cases), mean age 64.8 years (of whom 23 had a baseline deformity), and 196 controls, mean age 63.9 years (of whom 60 had a baseline deformity), were studied. There were strong correlations between the domain scores for each of the three instruments. There was no statistically significant difference in any of the domain scores between cases and those controls with a prevalent deformity. However, compared with the controls without a prevalent deformity the cases had significantly impaired quality of life as determined using the total QUALEFFO score (38.2 vs 33.7), the physical component score of the SF-12 (39.9 vs 43.7) and the health status score of the EQ-5D (62.3 vs 69.9). When the analysis was repeated after stratification of the cases by baseline deformity status (i.e., cases with and without a prevalent deformity at baseline), cases with a prevalent deformity had impaired quality of life compared with their matched controls, both with and without a prevalent deformity. In contrast there was no significant difference in quality of life among the cases without a prevalent deformity and either control group. Conclusion: In this population-based study a recent vertebral fracture was associated with impairment in quality of life, though this was mainly among those who had sustained a previous vertebral deformity.

Keywords Health impact - Osteoporosis - Quality of life - Vertebral fracture

\section{Introduction}

Vertebral fractures are linked with a variety of adverse health consequences including back pain and disability $[1,2,3,4]$, however, until recently, relatively little was known about the impact of vertebral fracture on healthrelated quality of life (HRQoL) either in clinic-based or population samples.

Health-related quality of life may be assessed using generic or disease-specific instruments. Generic instruments (e.g., Short Form 36 (SF-36) and Nottingham health profile (NHP)) can be used to assess quality of life across a range of disease states; however, they lack specificity for individual diseases. Disease-specific instruments have greater face and content validity for individual diseases than generic instruments. Several such instruments have been developed for use in assessing quality of life in osteoporosis, including the QUALEFFO, which was developed by a working group of the European Foundation for Osteoporosis [5]. The questionnaire comprises 41 questions organized into 5 domains: pain, physical function, social function, general health perception, and mental function. In a validation study, it showed good test-retest reliability and internal consistency, and discriminated between patients with clinically apparent vertebral fracture and control subjects [6].
Less than one third of patients with radiographic vertebral deformities, however, come to clinical attention, and therefore the impact of radiographic vertebral deformity on HRQoL in population samples is unclear $[7,8]$. Such data are important in order to characterize the overall health impact linked with vertebral fracture and indeed osteoporosis in general. Using participants in a population-based study of osteoporosis - the European Prospective Osteoporosis Study - we undertook a study with the aim of determining the impact of a recent vertebral fracture on HRQoL.

\section{Materials and methods}

\section{Subjects}

The subjects who took part in this study were recruited from those who had participated in a screening survey of vertebral osteoporosis - the European Vertebral Osteoporosis Study (EVOS). The detailed methods of this study are reported elsewhere [9]. In brief, subjects were recruited from population-based registers in 36 centers. Stratified random sampling was used with the aim of recruiting in each center, a target number of 50 subjects in each of six 5-year age and sex bands: 50-54, 55-59, $60-64,65-69,70-75$, and 75 years and over. Subjects were invited to attend for lateral radiographs of the thoracic and lumbar spine. In total, 15,570 men and women aged 50-79 years had baseline spinal radiographs performed. Subjects at 29 centers were invited to attend for a repeat spinal radiograph a mean of 3.8 years following the baseline examination. Both baseline and follow-up radiographs were performed using a standard protocol. For a variety of reasons including resource constraints only a proportion (approximately 50\%) of the 14,011 men and women who had baseline spinal films at these centers had a second radiograph performed. However, in a limited analysis the impact of nonparticipation on the incidence of vertebral fractures was small [10].

\section{Radiographic assessment}

Both baseline and follow-up spinal radiographs were evaluated morphometrically in a single center (Berlin). Prevalent deformities at baseline were defined morphometrically using the McCloskey-Kanis algorithm [11]. Incident vertebral fractures were classified both by qualitative (clinical) radiologist assessment and morphometrically. The radiologist (D.F.) reviewed paired sets of films in which any of the individual vertebral height ratios $(\mathrm{Ha} / \mathrm{Hp}$ or $\mathrm{Hm} / \mathrm{Hp})$ was less than 0.75 or if there had been a change in these ratios of $15 \%$ or more between films. At this assessment point placement was revised if it was felt necessary, and the radiologist made a clinical (qualitative) assessment about whether an incident vertebral fracture was present. The morphometric method used required a vertebra to satisfy criteria for a McCloskey-Kanis deformity on the second radiograph and, in addition show a change (between films) in either anterior, mid or posterior vertebral height of at least $20 \%$ (with the reduction in height being at least $4 \mathrm{~mm}$ ) [12].

\section{Quality of life study}

Twelve EPOS centers from seven countries (Czech Republic, Hungary, Italy, Slovakia, Spain, Sweden, UK) agreed to participate in the quality of life study. In total, 3,205 men and 
women, mean age 63.2 years, from these centers had paired spinal radiographs performed. There were 121 subjects with either morphometric or qualitative incident fracture (incidence in men, 4.8/1,000 person-years; in women, 12.2/1,000 person-years). In the quality of life study, "cases" were those subjects who had sustained an incident vertebral fracture based on either the radiologist's assessment or the morphometric criteria. For each case, three controls were selected as those next closest in age within each gender and center: one with a prevalent deformity at the baseline survey (control-P), and two without a prevalent deformity at the baseline survey (control-NP). Subjects who agreed to take part completed a HRQoL questionnaire (see below). The questionnaire was interviewer-assisted except in the UK centers where a postal survey was performed after validating the postal method in this setting [13]. Because of differences in time to complete the follow-up phase, there were between-center differences in the time between the second radiograph and the quality of life survey (median time 1.9 years). Within each center, however, both cases and controls were contacted at approximately the same time.

\section{HRQoL questionnaire}

The questionnaire included three quality of life instruments (in order): a generic QoL instrument, Short Form 12 (SF-12); a preference-based instrument, EQ-5D (formerly known as EuroQoL), and a disease-specific instrument, the QUALEFFO. For each center the appropriate language versions of these questionnaires were available and used, except in Budapest, where only QUALEFFO was available. Instruments were scored according to the algorithms provided by their development team $[14,15,16]$. The SF-12 summary health scales were scored using US standard weights since country-specific weights were not available for all countries. The SF-12 physical component summary scale (PCS) and mental component summary scale (MCS) are scored so that increasing values represent better HRQoL. The five questions from the EQ-5D were used to generate a weighted health utility index, based on tables of values derived from a UK general population [17]. Dolan used the time tradeoff (TTO) method to value health states. TTO valuations for a subset of EQ-5D states were elicited from more than 3,000 subjects. From these responses "tariff" values (on a scale in which 1 and 0 represent full health and death, respectively) were estimated for all 243 EQ-5D states. The EQ-5D health status VAS scale was scored as a single number that ranged from 0 (representing poor health) to 100 (representing good health). The QUALEFFO domain scores were transformed linearly to a $100-$ point scale with 0 representing the best HRQoL and 100 the worst HRQoL. The total score for the QUALEFFO was calculated in two ways: total score 1 being the average of the transformed domain scores, and total score 2 being the average of scores for all nonmissing individual questions.

\section{Statistical methods}

Spearman correlation coefficients were used to determine the association between the individual domains and total scores of the QUALEFFO, the two SF-12 summary scales, the five EQ-5D domains, and the EQ-5D health utility and health status scores. Comparisons between the cases and matched controls was undertaken using the signed rank test - a nonparametric paired test. The analysis was performed initially by comparing the cases with each of the two control groups (those with a prevalent deformity, control-P, and those without, control-NP). Subsequently the analysis was repeated after stratifying the cases into those with and those without a prevalent vertebral deformity (case-P and case-NP, respectively). These were then compared with their matched controls (control-P and control-NP).

\section{Results}

\section{Subjects}

Seventy-three subjects (57 women and 16 men) with an incident vertebral fracture (mean age 64.8 years), and 196 controls ( 151 women and 45 men) without an incident fracture (mean age 63.9 years) took part in the study. Agreement between the morphometric and qualitative definitions of incident vertebral fracture was good $(\kappa=0.82)$. Twenty-three $(32 \%)$ of the cases and 60 $(31 \%)$ of the controls had evidence of a prevalent vertebral deformity.

\section{Correlation between disease-specific and generic instruments}

Correlations between the domains of the QUALEFFO and the generic health measures, EQ-5D and the SF-12, are shown in Table 1. For all domains and the total QUALEFFO score, there were moderate correlations with the two generic instruments. Correlation coefficients were higher between corresponding domains for these instruments. Thus there were strong correlations between the physical function domain of the QUALEFFO and the PCS of the SF-12 $(r=-0.81)$ and with the mobility score of the EQ-5D $(r=0.69)$, and also usual activities $(r=0.69)$. The correlation between the mental function domain of the QUALEFFO and the MCS of the SF-12 was -0.64 .

\section{Impact of vertebral fracture on HRQoL}

The mean scores for the component domains of the QUALEFFO, EQ-5D, and SF-12, for the cases and the corresponding matched controls are presented in Table 2. Compared with the controls without a prevalent deformity at baseline (control-NP), the cases had poorer health as assessed using all domains of all three instruments, though the differences were statistically significant for the social function, general health, mental function, and total scores of the QUALEFFO, the physical function score of the SF-12, and mobility, pain/ discomfort, health utility, and health status of the EQ$5 \mathrm{D}$. When compared with those controls with a prevalent deformity at baseline for the QUALEFFO the differences were smaller and none attained conventional levels of statistical significance.

\section{Case stratification by baseline vertebral deformity}

The mean scores for each of the domains of the QUALEFFO, SF-12, and EQ-5D and their respective matched controls after stratification by baseline deformity status are presented in Tables 3 and 4 . The mean 
Table 1 Correlation between QUALEFFO (domain and total scores), and SF-12 and EQ-5D. Total score 1 average of domain scores, Total score 2 average of scores for individual questions

\begin{tabular}{|c|c|c|c|c|c|c|c|}
\hline & \multicolumn{7}{|c|}{ QUALEFFO $^{\mathrm{a}}$} \\
\hline & Pain & Physical function & Social function & General health & Mental function & Total score 1 & Total score 2 \\
\hline \multicolumn{8}{|l|}{$\mathrm{SF}-12^{\mathrm{a}}$} \\
\hline MCS & -0.42 & -0.53 & -0.48 & -0.54 & -0.64 & -0.61 & -0.62 \\
\hline PCS & -0.52 & -0.81 & -0.68 & -0.74 & -0.6 & -0.81 & -0.82 \\
\hline \multicolumn{8}{|l|}{ EQ-5D ${ }^{\mathrm{a}}$} \\
\hline Mobility & 0.4 & 0.69 & 0.59 & 0.58 & 0.5 & 0.66 & 0.68 \\
\hline Self-care & 0.25 & 0.51 & 0.46 & 0.43 & 0.34 & 0.46 & 0.48 \\
\hline Usual activities & 0.37 & 0.69 & 0.54 & 0.6 & 0.57 & 0.65 & 0.67 \\
\hline Pain/discomfort & 0.59 & 0.64 & 0.5 & 0.51 & 0.48 & 0.65 & 0.65 \\
\hline Anxiety/depression & 0.34 & 0.36 & 0.31 & 0.33 & 0.53 & 0.43 & 0.43 \\
\hline Health utility ${ }^{\mathrm{a}}$ & -0.57 & -0.8 & -0.67 & -0.66 & -0.65 & -0.79 & -0.81 \\
\hline Health status ${ }^{\mathrm{a}}$ & -0.49 & -0.68 & -0.54 & -0.71 & -0.51 & -0.7 & -0.69 \\
\hline
\end{tabular}

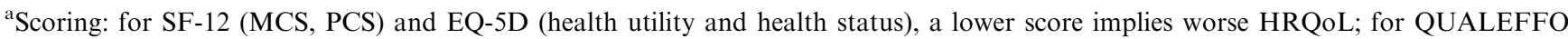
domains and EQ-5D (mobility, self-care, usual activities, pain/discomfort, and anxiety/depression), a lower score implies better HRQoL

scores for the controls are different in these tables because the controls were individually matched to the cases with incident deformities who did not have (case-NP; Table 3) or did have a baseline prevalent deformity (case-P, Table 4).

Overall there was no statistically significant difference between the cases without a prevalent deformity and either control group for any of the quality of life domains, apart from general health which was slightly worse in the controls with a prevalent deformity than the cases (see Table 3). In contrast, cases with a prevalent deformity had significant impairments in health compared with controls without a prevalent deformity for all domains except the self-care and anxiety depression of

Table 2 Mean domain scores for QUALEFFO, EQ-5D, and SF12 in all cases and their matched controls (with and without a baseline deformity).Case incident fracture either morphometrically or by radiologist,control- $P$ control with baseline prevalent defor- the EQ-5D (see Table 4). Compared with the controls with a prevalent deformity, cases with a prevalent deformity had impairments in quality of life for all domains of the QUALEFFO (except pain and physical function), both domains of the SF-12, and the usual activity domain of EQ-5D (see Table 4).

\section{Discussion}

In this analysis, subjects with a recent vertebral fracture had impairment in health-related quality of life as assessed using the QUALEFFO, SF-12, and EQ-5D compared with those without evidence of vertebral

mity, control-NP control without baseline prevalent deformity. Total score 1 average of domain scores, Total score 2 average of scores for individual questions

Control-P $\quad p$ Value

$(n=60)$

Case vs control-NP

Case vs control-P

QUALEFFO

$\begin{array}{cc}31.0(28.8) & 27.6(27.5) \\ 22.5(17.6) & 19.8(19.5) \\ 44.0(26.4) & 37.2(28.2) \\ 55.4(21.7) & 49.1(23.3) \\ 38.6(16.7) & 34.8(19.4) \\ 38.2(17.7) & 33.7(19.5) \\ 33.1(16.6) & 29.0(18.9) \\ & \\ 39.9(9.8) & 43.7(11.1) \\ 47.2(9.9) & 49.1(10.9) \\ 1.6(0.5) & 1.4(0.5) \\ 1.2(0.5) & 1.1(0.3) \\ 1.5(0.6) & 1.4(0.6) \\ 1.8(0.6) & 1.7(0.5) \\ 1.4(0.6) & 1.4(0.6) \\ 76.7(19.3) & 82.5(16.7) \\ 62.3(20.6) & 69.9(21.5)\end{array}$

$29.9(28.1)$
$20.2(18.0)$
$42.2(31.3)$
$54.9(22.5)$
$36.3(17.1)$
$36.6(18.6)$
$30.7(17.4)$

0.25

0.13

0.01

0.02

0.04

0.02

0.02

$41.2(11.3)$

$50.8(10.8)$

0.01

0.09

$1.5(0.5)$

$1.2(0.4)$

$1.3(0.5)$

$1.8(0.6)$

$1.3(0.5)$

80.1 (18.7)

66.3 (20.2)

$<0.01$

0.12

0.35

0.01

0.35

$<0.01$

$<0.01$

0.89

0.54

0.92

0.92

0.65

0.77

0.63

0.75

0.05

0.85

1

0.16

0.8

0.75

0.37

0.31

a Scoring: for SF-12 (MCS, PCS) and EQ-5D (health utility and health status), a lower score implies worse HRQoL; for QUALEFFO domains and EQ-5D (mobility, self-care, usual activities, pain/discomfort, and anxiety/depression), a lower score implies better HRQoL 
Table 3 Mean domain scores for QUALEFFO, EQ-5D, SF-12 in cases without a baseline deformity and their matched controls (with and without a baseline deformity). Case incident fracture either morphometrically or by radiologist, control-P control with baseline prevalent deformity, control-NP control without baseline prevalent deformity. Total score 1 average of domain scores, Total score 2 average of scores for individual questions

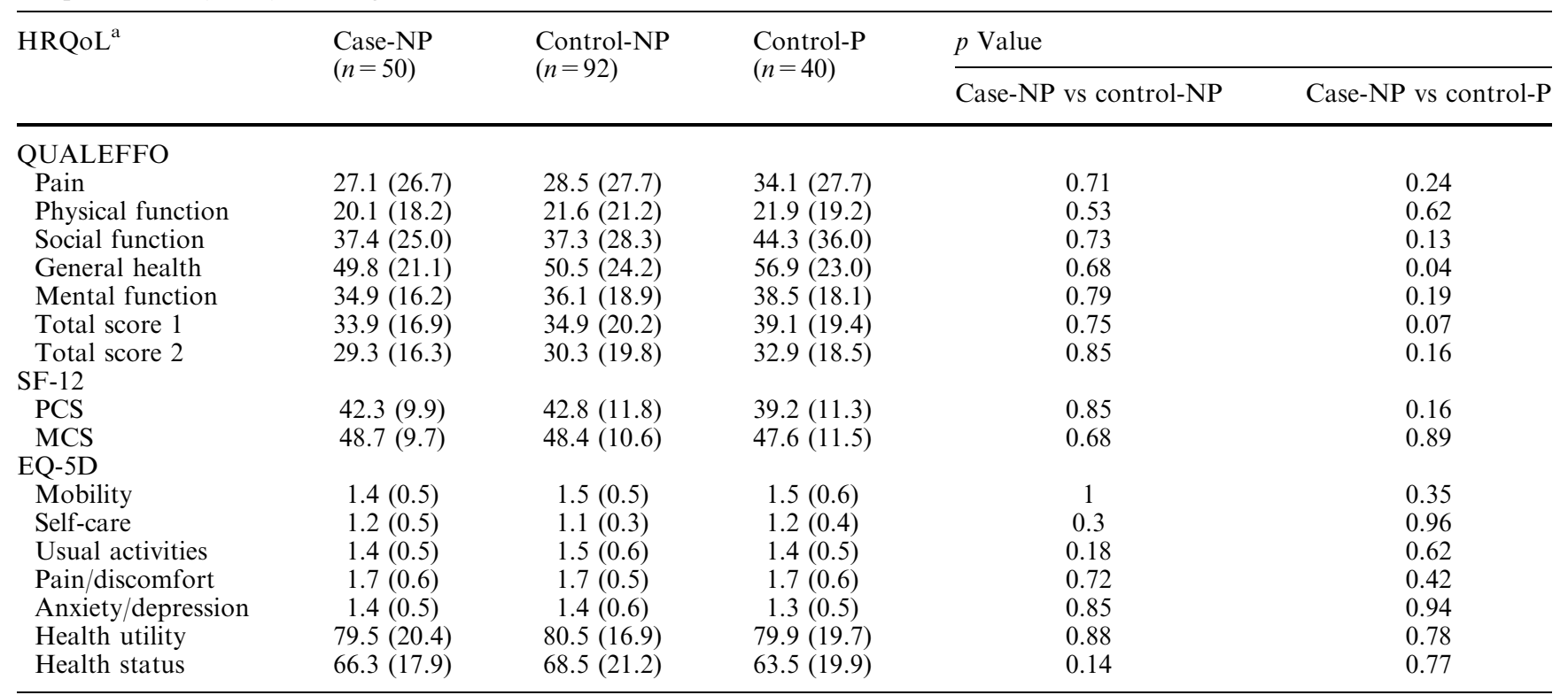

${ }^{a}$ Scoring: for SF12 (MCS, PCS) and EQ-5D (health utility and health status), a lower score implies worse HRQoL; for QUALEFFO domains and EQ-5D (mobility, self-care, usual activities, pain/discomfort, and anxiety/depression), a lower score implies better HRQoL

fracture. The adverse health impact was most marked among those with a recent fracture who had a preexisting vertebral deformity, suggesting that the effect on quality of life of multiple fractures is cumulative.

Both disease-specific and generic instruments have been used to assess quality of life in patients with

Table 4 Mean domain scores for QUALEFFO, EQ-5D, and SF12 in cases with a baseline deformity and their matched controls (with and without a baseline deformity). Case Incident fracture either morphometrically or by radiologist, control-P control with vertebral osteoporosis, though mainly in the setting of clinical trials or clinically based studies $[6,16,18,19]$. Indeed, assessment of health-related quality of life has become an important outcome criterion in clinical trials of vertebral osteoporosis in addition to assessment of BMD and fracture incidence. In our study we used two

baseline prevalent deformity, control-NP control without baseline prevalent deformity. Total score 1 average of domain scores, Total score 2 average of scores for individual questions

\begin{tabular}{|c|c|c|c|c|c|}
\hline \multirow[t]{2}{*}{$\mathrm{HRQoL}^{\mathrm{a}}$} & \multirow{2}{*}{$\begin{array}{l}\text { Case-P } \\
(n=23)\end{array}$} & \multirow{2}{*}{$\begin{array}{l}\text { Control-NP } \\
(n=44)\end{array}$} & \multirow{2}{*}{$\begin{array}{l}\text { Control-P } \\
(n=20)\end{array}$} & \multicolumn{2}{|l|}{$p$ Value } \\
\hline & & & & Case-P vs control-NP & Case-P vs control-P \\
\hline \multicolumn{6}{|l|}{ QUALEFFO } \\
\hline Pain & $40.0(32.0)$ & $25.7(27.3)$ & $22.0(27.8)$ & 0.01 & 0.06 \\
\hline Physical function & $27.7(15.5)$ & $16.1(14.8)$ & $16.8(15.3)$ & $<0.001$ & 0.08 \\
\hline Social function & $61.2(22.4)$ & $36.8(28.4)$ & $37.7(18.5)$ & $<0.001$ & 0.04 \\
\hline General health & $67.4(18.3)$ & $46.4(21.4)$ & $50.8(21.4)$ & $<0.001$ & 0.01 \\
\hline Mental function & $46.5(15.1)$ & $32.0(20.1)$ & 31.9 (14.2) & $<0.001$ & 0.01 \\
\hline Total score 1 & $47.6(15.7)$ & $31.4(17.9)$ & $31.5(16.2)$ & $<0.001$ & 0.01 \\
\hline Total score 2 & $41.5(14.4)$ & $26.3(16.8)$ & $26.2(14.3)$ & $<0.001$ & 0.01 \\
\hline \multicolumn{6}{|l|}{ SF-12 } \\
\hline PCS & $34.6(7.3)$ & $45.6(9.5)$ & $44.7(10.5)$ & $<0.001$ & 0.02 \\
\hline MCS & $43.9(9.6)$ & $50.5(11.6)$ & $56.8(6.2)$ & 0.03 & 0.001 \\
\hline \multicolumn{6}{|l|}{ EQ-5D } \\
\hline Mobility & $1.8(0.5)$ & $1.3(0.4)$ & $1.5(0.5)$ & $<0.001$ & 0.1 \\
\hline Self-care & $1.2(0.5)$ & $1.1(0.3)$ & $1.2(0.5)$ & 0.18 & 0.95 \\
\hline Usual activities & $1.7(0.6)$ & $1.3(0.5)$ & $1.2(0.4)$ & 0.003 & 0.005 \\
\hline Pain/discomfort & $2.1(0.6)$ & $1.6(0.6)$ & $1.8(0.5)$ & $<0.001$ & 0.13 \\
\hline Anxiety/depression & $1.4(0.6)$ & $1.3(0.5)$ & $1.3(0.5)$ & 0.2 & 0.65 \\
\hline Health utility & $70.7(15.6)$ & $86.7(15.7)$ & $80.6(17.2)$ & $<0.001$ & 0.05 \\
\hline Health status & $53.4(23.9)$ & $72.6(22.1)$ & $71.7(20.1)$ & $<0.001$ & 0.05 \\
\hline
\end{tabular}

a Scoring: for SF-12 (MCS, PCS) and EQ-5D (health utility and health status), a lower score implies worse HRQoL; for QUALEFFO domains and EQ-5D (mobility, self-care, usual activities, pain/discomfort, and anxiety/depression), a lower score implies better HRQoL 
generic instruments-SF-12 and EQ-5D (preferencebased) - and a disease-specific instrument-QUALEFFO. Our reason for using QUALEFFO instead of other available instruments was its good performance when compared with other instruments [6] and its availability in the different European languages in which its use was intended [20,21,22].

In a validation study using QUALEFFO, Lips et al. compared patients coming to clinical attention with controls without back pain or vertebral fracture using the QUALEFFO and the SF-36 [6]. Those with vertebral fracture were significantly more likely to report impairment both overall and in each domain of the QUALEFFO (social, mental, general health, physical and general health). In a recent study of 751 European women participating in a clinical trial (the MORE study) and using QUALEFFO, assessment at baseline showed that women with a prevalent deformity had decreased overall health-related quality of life compared with those without, and this was true for all domains except mental function [16]. Furthermore, the adverse health impact increased with increasing number of fractures.

Our study extends these findings to the population setting and confirms the adverse health impact on quality of life of radiographic vertebral fracture. There were, however, some differences between the findings of our study and previous studies in relation to the individual domain scores of the instrument. Thus in contrast to the other studies, although the QUALEFFO domain scores for pain and physical function were higher (indicating worse health), they were not statistically significant from the nonfracture controls. This may reflect the greater prevalence of symptoms in those with clinically apparent fractures. In contrast, we observed impairment in mental function, while no difference was observed in the MORE study between those with and without fracture. It is possible this may be because of selection factors related to participation in a clinical trial or that agreeing to participate in a clinical trial may reduce previous anxiety and psychological distress [23]. However, the numbers in our study were relatively small and some caution is required in interpretation.

One of the main limitations of disease-specific instruments is that they can not be used to compare the impairment in quality of life related to osteoporosis with that due to other diseases for which a generic quality of life instrument is required. Generic instruments have been used previously to assess quality of life in vertebral osteoporosis, including the SF-36, Nottingham health profile (NHP), and EQ-5D [6, 16, 18, 24]. However, as with the disease-specific instruments, most studies have been undertaken in the clinical setting with few data from population-based studies. Most suggest that vertebral osteoporosis is linked with impairment in quality of life $[6,16,18,24]$. In our study we found significant impairment in the health status and health utility scores of the EQ-5D with significant differences also in the mobility and pain/discomfort questions, and also in the physical component score of the SF-12.
We found no important difference in health-related quality of life between our cases with recent fracture and controls with a prevalent vertebral deformity. This is perhaps not surprising given that both had already sustained at least one vertebral fracture. Differences in time between the baseline and quality of life survey may have reduced the impact of a recent fracture, which perhaps might have been expected - at least in the short term-to lead to greater impairment in quality of life compared with longer standing deformities. Further research using shorter time windows between radiographs and assessment of quality of life is required to explore this.

In subgroup analysis, one of the interesting findings from our study was the observation that the impact on health-related quality of life was more marked among those with a recent vertebral fracture provided they had already sustained a previous vertebral deformity. Differences in HRQoL were greater when these subjects were compared with controls without evidence of a preexisting vertebral deformity. This is clearly in part due to an effect of vertebral fracture number ("cases" with preexisting deformity having at least two fractures), with adverse health impact known to increase with increasing number of fractures [16]. However, there were also significant differences when these cases were compared with the controls with prevalent deformity. Taken together with the observation that those with recent fracture and no preexisting deformity did not differ from the controls without any fracture this suggests perhaps that for radiographic fractures, it is the second fracture which results in deterioration in health-related quality of life. Given the study design it was not possible to test the hypothesis directly.

There are methodological limitations to be considered in interpreting these data. In EPOS the occurrence of incident vertebral fractures was based on assessment of duplicate spinal radiographs undertaken on average 3.8 years apart and the quality of life study was performed a median of 1.9 years following the repeat spinal radiograph. Thus our results refer to the impact of recent fractures which occurred several years prior to assessment of quality of life. It is possible that the effect of the delay may have been to attenuate the health impact of the "incident" (recent) fractures and to reduce the likelihood of finding differences between those with and without "incident" vertebral fracture. In the SF-12 and EQ-5D, the questions about pain did not specify site, though in the QUALEFFO, there were questions specifically about back pain. For all three instruments, however, the focus was on current or recent pain. Episodes of back pain which occurred prior to this time and which had resolved would not therefore be captured by any of the instruments.

Our data was based on a questionnaire instrument translated into five European languages. It is possible that differences in the interpretation of these questions may have resulted in differential responses in different participating centers; however, given that the cases and 
controls were matched by center, any between-center differences in response are unlikely to have influenced the main findings.

In summary in this population-based study, individuals with a recent vertebral fracture had impaired quality of life compared with those without vertebral fracture. The adverse effect on health-related quality of life was more marked in those who had already sustained a preexisting prevalent deformity. Assessment of health-related quality of life is important in population studies of vertebral osteoporosis, and the adverse health impact should be considered in determining the overall impact of the disease on society.

Acknowledgements The study was financially supported by European Union Concerted Action Grants under Biomed-1 (BMH 1CT 931448 and 920182), and also EU grants C1PDCT925102, ERBC1PDCT 930105 \& 940229. The central coordination was also supported by the UK Arthritis Research Campaign, the Medical Research Council (G9321536), and the European Foundation for Osteoporosis and Bone Disease. Also a grant from Eli Lilly and Company. The EU's PECO program linked to Biomed-1 funded in part the participation of the Budapest, Warsaw, Prague, Piestany, Szczecin, and Moscow centers. Data collection in Cambridge was supported by a HSR grant from the Anglia and Oxford region. The central X-ray evaluation was generously sponsored by the Bundesministerium fur Forschung and Technologie, Germany. Individual centers acknowledge the receipt of locally acquired support for their data collection. We would like to thank the following individuals: Rita Smith, Aberdeen, UK; Uday Bhonsle, Anna Martin, Judith Walton, and Bridget Wardley-Smith, Cambridge and Harrow, UK; Mrs Joanna Parsons, Truro, UK; and J. Bernardino Diaz Lopez and Ana Rodriguez Rebollar, Oviedo, Spain. We would also like to thank the individuals who took part in the study and the many individuals who helped access our population samples.

\section{References}

1. Leidig G, Minne HW, Sauer P et al (1990) A study of complaints and their relation to vertebral destruction in patients with osteoporosis. Bone Miner 8:217-229

2. Ettinger B, Black DM, Nevitt MC et al (1992) Contribution of vertebral deformities to chronic back pain and disability. J Bone Miner Res 7:449-456

3. Matthis C, Weber U, O'Neill TW, Raspe H and the European Vertebral Osteoporosis Study Group (1998) Health impact associated with vertebral deformities: results from the European Vertebral Osteoporosis Study (EVOS). Osteoporos Int $8: 364-372$

4. Nevitt MC, Ettinger B, Black DM et al (1998) The association of radiographically detected vertebral fractures with back pain and function: a prospective study. Ann Intern Med 128:793800

5. Lips P, Cooper C, Agnusdei D et al (1997) Quality of life as outcome in the treatment of osteoporosis: the development of a questionnaire for quality of life by the European Foundation for Osteoporosis. Osteoporos Int 7:36-38

6. Lips P, Cooper C, Agnusdei D et al (1999) Quality of life in patients with vertebral fractures: validation of the Quality of Life Questionnaire of the European Foundation for Osteoporosis (QUALEFFO). Osteoporos Int 10:150-160
7. Cooper C, Atkinson EJ, O'Fallon WM, Melton LJ III (1992) Incidence of clinically diagnosed vertebral fractures: a population based study in Rochester, Minnesota, 1985-1989. J Bone Miner Res 7:221-227

8. van Staa TP, Dennison EM, Leufkens HGM, Cooper C (2001) Epidemiology of fractures in England and Wales. Bone 29:517522

9. O'Neill TW, Felsenberg D, Varlow J et al (1996) The prevalence of vertebral deformity in European men and women: the European Vertebral Osteoporosis Study. J Bone Miner Res 11:1010-1017

10. European Prospective Osteoporosis Study (EPOS) Group (2002) Incidence of vertebral fracture in Europe: results from the European Prospective Osteoporosis Study (EPOS). J Bone Miner Res 17:716-724

11. McCloskey EV, Spector TD, Eyres KS, Fern ED, O’Rourke N, Vasikaran S, Kanis JA (1993) The assessment of vertebral deformity: a method for use in population studies and clinical trials. Osteoporos Int 3:138-147

12. Lunt M, Ismail AA, Felsenberg D, Cooper C, Kanis JA, Reeve $\mathbf{J}$ et al (2002) Defining incident vertebral deformities in population studies: a comparison of morphometric criteria. Osteoporos Int 13:809-815

13. Murrell P, Todd CJ, Martin A, Walton J, Lips P, Reeve J, on behalf of the working party for quality of life of the International Osteoporosis Foundation (2001) Postal administration compared with nurse-supported administration of the QUALEFFO-41 in a population sample: comparison of results and assessment of psychometric properties. Osteoporos Int 12:672679

14. EuroQol Group (1996) EQ-5D user guide. EuroQol Group, Rotterdam, The Netherlands

15. Ware JE, Kosinski M, Keller SD (1998) SF-12: how to score the SF-12 physical and mental health summary scales, 3rd edn. Quality Metric, Lincoln, RI

16. Oleksik A, Lips P, Dawson A, Minshall ME, Shen W, Cooper C, Kanis J (2000) Health related quality of life in postmenopausal women with low BMD with or without prevalent vertebral fractures. J Bone Miner Res 15:1384-1392

17. Dolan P (1997) Modelling valuations for EuroQol health states. Med Care 35:1095-1108

18. Scane AC, Sutcliffe AM, Francis RM (1994) The sequelae of vertebral crush fractures in men. Osteoporos Int 4:89-92

19. Silverman SL, Minshall ME, Shen W, Harper KD, Xie S, on behalf of the Health-Related Quality of Life Subgroup of the Multiple Outcomes of Raloxifene Evaluation Study (2001) The relationship of health related quality of life to prevalent and incident vertebral fractures in postmenopausal women with osteoporosis. Arthritis Rheum 44:2611-2619

20. Osteoporosis Quality of Life Study Group (1997) Measuring quality of life in women with osteoporosis. Osteoporos Int 7:478-487

21. Lydick E, Zimmerman SI, Yawn B et al (1997) Development and validation of a discriminative quality of life questionnaire for osteoporosis (the OPTQol). J Bone Miner Res 12:456-463

22. Randell AG, Bhalerao N, Nguyen TV, Sambrook PN, Eisman JA, Silverman SL (1998) Quality of life in osteoporosis: reliability, consistency and validity of the Osteoporosis Assessment Questionnaire. J Rheumatol 25:1171-1179

23. Kessenich CR, Guyatt GH, Rosen CJ (1998) Health-related quality of life and participation in osteoporosis clinical trials. Calcif Tissue Int 62:189-192

24. Hall SE, Criddle RA, Comito TL, Prince RL (1999) A case control study of quality of life and functional impairment in women with long standing vertebral osteoporotic fracture. Osteoporos Int 9:508-515 